\title{
Resiliencia en mujeres universitarias en Norte de Santander, Colombia
}

\author{
Mawency Vergel-Ortega, Ph. D. ${ }^{a}$ \\ Universidad Francisco de Paula Santander, Colombia \\ Carlos Sebastián Gómez-Vergel ${ }^{\mathrm{b}}$ \\ Universidad de Los Andes, Colombia \\ Henry de Jesús Gallardo-Pérez, Ph. D. ${ }^{c}$ \\ Universidad Francisco de Paula Santander, Colombia
}

mawencyvergel@ufps.edu.co

\section{Resumen (analítico)}

La investigación tuvo por objeto analizar la resiliencia de estudiantes de posgrados de género femenino, en universidades públicas del Norte de Santander. La investigación tuvo un enfoque cuantitativo de tipo correlacional, método analítico descriptivo, encuesta y test de resilience scale, el cual fue aplicado a 150 estudiantes con edades entre los 27 y 45 años. Como resultado se establece que la resiliencia se asocia con fortaleza de carácter. Las características de las mujeres con alto nivel de resiliencia fueron centradas en el saber vivir con categoría primaria calidad de vida, en el saber conocimiento, en saber hacer y adquirir mayor destreza en conocimientos y automotivarse para saber estar. Se concluye que la escala de resiliencia ajustada con los factores planificación y empatía presenta evidencias de validez con relación a su estructura interna y fiabilidad de 0.87 .

\section{Palabras clave}

Mujer, universidad pública, calidad de vida, motivación.

\section{Thesauro}

Tesauro de Ciencias Sociales de la Unesco.

\section{Para citar este artículo}

Vergel-Ortega, M., Gómez-Vergel, C. S., \& Gallardo-Pérez, H. de J. (2021). Resiliencia en mujeres universitarias en Norte de Santander, Colombia. Revista Latinoamericana de Ciencias Sociales, Niñez y Juventud, 19(3), 1-19. https://dx.doi.org/10.11600/rlcsnj.19.3.4590

\section{Historial}

Recibido: 19.08 .2020

Aceptado: 25.01.2021

Publicado: 30.07 .2021

\section{Información artículo}

El artículo hace parte del desarrollo de la primera fase de la investigación denominada Percepción de la ciudadanía del servidor público en Norte de Santander, hacia la construcción de paz. Un modelo estructural, de febrero 2019 a Diciembre de 2019, en el cual se analiza, entre otros factores, la resiliencia en diferentes grupos de actores. El proyecto contó con aprobación y financiación del Fondo de Investigaciones Universitarias de la Universidad Francisco de Paula Santander, mediante contrato Finu 035-2019 de septiembre de 2019; no obstante, el trabajo se inició desde febrero de 2019. Área: ciencias sociales; subárea: estudios de género y estadística 


\section{Resilience in University Women in Norte de Santander-Colombia}

Abstract (analytical)

The purpose of the study was to analyze the resilience of female graduate students in public universities in Norte de Santander. The research used a quantitative approach of a correlational type and a descriptive analytical method. The study involved the application of a survey and resilience scale test to 150 students aged between 27 and 45 years old. The results showed that resilience is associated with strength of character. The characteristics of female university students with a high level of resilience were centered on quality of life, obtaining knowledge, how to apply this knowledge, acquiring further, self-motivation and knowing how to be. Conclusion: The resilience scale, adjusted to accommodate the factors of planning and empathy, presents evidence of validity in relation to its internal structure and reliability of 0.87 .

Keywords

Woman, public university, quality of life, motivation.

\section{Resiliência em Mulheres Universitárias no Norte de Santander, Colômbia}

Resumo (analítico)

A investigação teve como objetivo analisar a resiliência de estudantes do sexo feminino de programas de pós-graduação em universidades públicas do Norte de Santander. A investigação teve uma abordagem quantitativa de tipo correlacional, método analítico descritivo, inquérito e teste de resilience scale que foi aplicado a 150 alunas com idade média entre os 27 e 45 anos. Resultado: a resiliência mostrou-se associada à força de carácter, as características das mulheres com elevado nível de resiliência centraram-se em saber viver com qualidade de vida de categoria primária, no saber conhecer, saber fazer e adquirir maior destreza nos conhecimentos, automotivadas a saber estar. Conclusão: a escala de resiliência ajustada com fatores de planeamento e empatia apresentou evidências de validade em relação à sua estrutura interna e fiabilidade de 0.87 .

Palavras-chave

Mulher, universidade pública, qualidade de vida, motivação.

\section{Información autores}

[a] Licenciada en Matemáticas y Física, Universidad Francisco de Paula Santander. Magíster en Educación, Universidad Pedagógica Experimental Libertador. Doctora en Educación, Universidad Pedagógica Experimental Libertador.

Posdoctora en Imaginarios y Representaciones Sociales, Universidad Pedagógica Experimental Libertador. Posdoctora del Programa de Investigación Postdoctoral en Ciencias Sociales, Niñez y Juventud, Centro de Estudios Avanzados en Niñez y Juventud del Cinde y la Universidad de Manizales. Profesora departamento de Matemáticas y Estadística, Universidad Francisco de Paula Santander, Colombia. iD 0000-0001-8285-2968. H5: 21. Correo electrónico: mawencyvergel@ufps.edu.co

[b] Universidad de los Andes, Colombia. Estudiante Ingeniería Electrónica. iD 0000-0002-6176-3613. H5: 6. Correo electrónico: cs.gomezv@uniandes.edu.co

[c] Licenciado en Matemáticas y Física, Universidad de Pamplona. Especialista en Estadística, Universidad Nacional de Colombia. Magíster en Economía, Universidad de los Andes. Doctor en Educación, Universidad Pedagógica Experimental Libertador. Profesor del departamento de Matemáticas y Estadística, Universidad Francisco de Paula Santander, Colombia. iD 0000-0003-4377-3903. H5: 6. Correo electrónico:

henrygallardo@ufps.edu.co 


\section{Introducción}

ـ a resiliencia es un constructo definido como la habilidad individual y medible pueda estar inmerso (Aguilar et al., 2019; Connor et al., 2003). Es considerada desde la psicología como conducta asumida por estudiantes para lograr tanto objetivos educativos como metas profesionales y personales. Es común encontrar como tendencia, un alto porcentaje de jóvenes que triunfan y cursan programas académicos universitarios, el alcanzar puntajes medios y altos en las escalas de resiliencia general (Manzano \& Ayala, 2013). Investigaciones señalan como factores relevantes al contexto académico asociados a la resiliencia la autoeficacia académica (Bender \& Ingram, 2018), asumir conductas resilientes que involucran el optimismo, la valoración, el apoyo externo y las expectativas realistas conformando una comunidad universitaria más adaptativa (DeRosier et al., 2013). Algunos investigadores indican su relación con factores sociodemográficos (Vergel-Ortega et al., 2016) y la salud, asociados a personalidad, familia (Salazar \& Vergel, 2018), apoyo social, depresión, ansiedad y estrés (Vergel-Ortega et al., 2015).

De otra parte, distintos estudios señalan la existencia de un currículo oculto en los programas educativos que reproduce estereotipos o representaciones hegemónicas de masculinidades y feminidades discriminatorias (Domínguez, 2016); así como la existencia de un discurso hegemónico escolar relativo a las relaciones de género que tiende a legitimar roles de femineidad y masculinidad tradicional y que son interpuestos tanto por profesores como por el alumnado (Sameroff \& Rosemblum, 2006). También el arraigo de valores androcéntricos en el profesorado, la escasez de formación en igualdad de género y opiniones de no actuar en instituciones porque en su opinión la igualdad de oportunidades entre hombres y mujeres es un hecho superado (DeRosier et al., 2013), la relación entre el rendimiento académico y el éxito escolar en estudiantes de educación superior encontrando que los estudiantes desarrollan habilidades emocionales de resiliencia que les permiten mejorar su calidad de vida (Santiago et al., 2020). 
La importancia de la investigación radica en determinar factores que se relacionan o asocian a la resiliencia en las mujeres estudiantes de posgrados en Norte de Santander (Colombia), a fin de generar estrategias pedagógicas que generen conciencia en las niñas de la región en torno a su futura formación profesional y al liderazgo que deben asumir en el país. Esto conlleva a formular la pregunta de investigación: ¿cómo analizar la resiliencia de estudiantes de postgrados de género femenino, en universidades públicas del Norte de Santander? Lo cual conlleva a una segunda pregunta: ¿es necesaria una escala para evaluar los factores de resiliencia en mujeres universitarias contextualizada en la región? Para responderlas, se analizan creencias, desafíos, eficacia, factores de protección a partir de diferentes escalas. Para el desarrollo de la investigación se toma como referencia la escala de resiliencia de Connor y Davidson (American Educational Research Association et al., 2014).

\section{Resiliencia}

La resiliencia se ha entendido como la resistencia de un cuerpo a su rotura, la capacidad de un material de recobrar su estado original tras someterse a presión o deformación (Fu et al., 2014); como la habilidad para adaptarse y restaurar el equilibrio a través de la autoconfianza, disciplina, autoestima y control (Khoshouei, 2009); o como los esfuerzos cognitivos y conductuales dirigidos a manejar las demandas internas o externas evaluadas como algo que agrava o excede los recursos de la persona (Benavente \& Quevedo, 2018). Los primeros estudios surgen en 1985 con niños que vivían experiencias adversas y que eran capaces de desarrollarse de forma normal y competente en la adultez (Vásquez et al., 2006) con variables protección, vulnerabilidad, comunidad y familia (Cicchetti \& Rogosch 2012). En 1974 surgen estudios en psicopatología en padres con trastornos mentales como etiología y resistencia al estrés con variable autoestima, así como estudios en el ámbito empresarial, para analizar dinámicas llevadas a cabo por entidades capaces de afrontar cambios una vez afectada su rentabilidad, afrontar riesgos y asimilar fracasos (Belykh, 2018). En el contexto de la resiliencia, el riesgo y la protección permanecen en constante movimiento; su identificación y dinámica conllevan a buscar salidas más allá del determinismo para abrirse camino a la creatividad y a la libertad (García-Vega, \& Domínguezde la Ossa, 2013).

La resiliencia desde la formación como proceso socializador de construcción de identidad y desarrollo personal ha permeado aproximaciones de modelos pedagógicos proactivos formados para la construcción de fortalezas internas (Garmezy, 1991), donde 
la resiliencia es una característica primordial para el éxito. Henderson y Milstein (2003) diseñaron dimensiones de afecto, expectativas, oportunidades de participación, vínculos prosociales, límites claros y habilidades para la vida donde quienes se forman sienten el ámbito escolar como ámbito afectivo, con sensaciones de pertenencia (Martínez et al., 2016), comunidad donde es un ser reconocido y esto le genera confianza; con resultado de autoánimo (Vergel-Ortega et al., 2014), eficacia, ser escuchado y puesto al servicio comunitario de sus conocimientos que le potencian sus relaciones e interacciones para comprender y participar en estados de políticas y reglas, con continua instrucción para integrar habilidades y adopción de buenas decisiones en el manejo de conflictos y sano estrés (Vergel-Ortega et al., 2018). Es posible desarrollar la resiliencia en cualquier situación que provoque sufrimiento o en cualquier experiencia que sea vivida como una adversidad (Henderson, 2016).

\section{Metodología}

La investigación se enmarca en el paradigma cuantitativo y se aborda desde un enfoque descriptivo analítico aplicando métodos tipo correlacional (Vergel-Ortega et al., 2018). Complementariamente se desarrolla desde un enfoque multimétodo (GallardoPérez et al., 2018) con apoyo cualitativo, fundamentado en la teoría de lo antropológico, específicamente en estudios de género.

La población objeto de estudio está constituida por mujeres matriculadas en programas de maestría en las universidades estatales de la región: Universidad Francisco de Paula Santander, Universidad de Pamplona y Escuela Superior de Administración Pública. La muestra se seleccionó mediante el método aleatorio estratificado con afijación proporcional, quedando conformada por 150 mujeres. Las participantes en el estudio tienen edad comprendida entre los 27 y 45 años, con un promedio de 34 y una desviación estándar de 4.12 años; sin embargo, el $70 \%$ está entre los 27 y 35 años. Con referencia al estado civil, se encuentra que el $20 \%$ son solteras, $17 \%$ están en unión libre, $52 \%$ son casadas y $11 \%$ viudas o separadas. Con respecto a la ubicación geográfica del lugar de trabajo, el 55\% trabaja en instituciones ubicadas en los municipios de Cúcuta, Pamplona, Los Patios y El Zulia y el $45 \%$ restante se localiza en otros municipios del departamento.

La información se recolectó mediante la aplicación de diferentes instrumentos. En una primera fase, se aplicó la escala de resiliencia de Connor y Davinson (2003) (Crespo 
et al., 2014), instrumento de evaluación multidimensional de la resiliencia compuesto por 32 ítems en escala Likert (Yárnoz-Yaben \& Comino-González, 2012), con cinco opciones de respuesta y cuyo índice de fiabilidad alfa de Conbrach fue de o.81 para las mujeres estudiantes de posgrado en el departamento de Norte de Santander. Se utilizó también, en una segunda fase, como segundo instrumento, el cuestionario de Fortalezas de carácter (Values in Action) (Vásquez, 2008). Este es inventario que consta de 240 ítems tipo Likert con cinco posibles respuestas cada uno ( 5 «muy parecido a mí», a 1 «nada parecido a mí»); este instrumento permite medir el grado que los estudiantes poseen en cada una de las 24 fortalezas ordenadas bajo 6 virtudes, con validez alta Kappa=0.85 (Cohen, 1992). Adicionalmente, en tercera fase, se realizó entrevista con un índice de Kappa=0.75, considerado válida (Chen, 2007), sobre el estilo de vida y las características de integrantes de familia paternos y maternos.

Tanto la información cuantitativa obtenida mediante la aplicación de las escalas de resiliencia referidas como la información cualitativa proveniente de la entrevista fueron procesadas mediante procedimientos de análisis de datos utilizando el software SPSS (Crespín, 2016). Para los datos cuantitativos se utilizó el análisis de ecuaciones estructurales (Avendaño et al. 2014), técnica que combina los modelos de regresión múltiple con métodos de análisis factorial y permite establecer la relación de dependencia entre las variables, así como evaluar las interrelaciones de dependencia al tiempo que incorpora los efectos del error de medida sobre los coeficientes estructurales (Escobedo et al. 2015). La información procedente de la entrevista fue codificada con el fin de establecer categorías de análisis que se incorporaron tanto en el procesamiento de datos como en el modelo estructural (Ruiz et al. 2010).

Para analizar los reactivos se establecieron medias aritméticas, desviaciones estándar, asimetría y curtosis, así como el estadístico de la prueba de Kolmogorov-Smirnov (Burns \& Anstey, 2010), junto con el nivel de significación con el fin de evaluar la normalidad de la distribución. Después se llevó a cabo un análisis factorial exploratorio mediante el método de componentes principales con rotación ortogonal varimax (Saris et al., 2009), basado en autovalores superiores a 1 , donde se tomaron en consideración las medidas de Kaiser-Meyer-Olkin (KMO) mayores a o.8o y la prueba de esfericidad de Bartlett en un nivel significativo. En la prueba de Levene para igualdad de varianzas para muestras independientes $\mathrm{p}=0.11>0.05$, por lo cual se acepta la igualdad de varianzas (Zinbarg et al., 2006). Se realizó análisis de test para la escala de resiliencia de Connor y Davidson a través del método de extracción WLSMV estimador que no asume variables distribuidas 
normalmente con base en la matriz de correlaciones policóricas (Cassidy, 2015). Se analizaron parámetros que afectaran la calidad del modelo (Asparouhov \& Muthén, 2009) y el modelo bifactor, postulando un factor general que explica la mayor variabilidad de ítems, coeficiente omega jerárquico general ( $\omega \mathrm{h}$ ) y de las dimensiones ( $\omega \mathrm{hs}$ ) (Fleming \& Merino, 2005), siendo sustancial cuando es $\geq 0.30$ (Baek et al., 2010). Se especificó la rotación geomin $(\varepsilon=0.05)$ para el modelo oblicuo y rotación target para el bifactor (Vizoso-Gómez, \& Arias-Gundín, 2018). En el modelo oblicuo fue interpretado con el índice de simplicidad factorial ISF (Ponce, 2015) para analizar el impacto de cargas factoriales secundarias donde, para valores mayores a 0.70 , fue considerado de simplicidad aceptable (McDonald \& Ho, 2002), lo que indicaría si el ítem recibe influencia significativa predominantemente de un factor, asimismo, varianza común explicada y porcentaje de correlaciones superior a 0.7 (Smits, 2015). Se analizó el ajuste estadístico de los modelos según el índice de ajuste comparativo (DiStefano et al., 2018) (CFI>0.9o), el límite superior del intervalo de confianza (IC) del índice de aproximación de la raíz de cuadrados medios del error (RMSEA<.10) (Baek et al., 2010) y la raíz cuadrada media residual ponderada (WRMR $<1$ ) (Schmidt, 2008). Se consideró la magnitud de las cargas factoriales mayor a 0.50 (Domínguez, 2018) y de las correlaciones interfactoriales $\phi<0.80$ (García-Portilla et al., 2017).

\section{Resultados}

La entrevista mostró perfiles de mujeres líderes, trabajadoras, con hogares establecidos, algunas cabezas de hogar, con deseo de superación, con y sin familia (o a 3 hijos), así como importancia dada al logro y no a las personas. Estas categorías fueron identificadas como emergentes, así como la presencia de violencia de género en hogares de niñez (30\%) con alta frecuencia en mujeres mayores de 35 años. De otra parte, un 20 \% manifestó que ha vivido situaciones en las cuales prima la preferencia a labor masculina en las instituciones en torno a roles directivos. 10 \% señaló inconformidad en evaluaciones, en particular donde son mujeres las evaluadoras y quienes otorgan mayor puntaje a los hombres. El $80 \%$ de quienes han logrado cargos en alta dirección han tenido un familiar como ejemplo a seguir en su futuro profesional. Las categorías identificadas en esta fase fueron: individualidades, cultura de machismo, ejemplo de vida y superación.

Las características de las mujeres con alto nivel de resiliencia fueron centradas en el saber vivir con categoría primaria calidad de vida centrada en la familia; así mismo en el 
saber ser (dimensión personal, actitud y comportamiento), conocer sus fortalezas y control de debilidades, no tener miedo a los cambios, analizar las situaciones, responder a tiempo a las adversidades, enfrentar los conflictos, ser sinceras, manejar emociones (pero después del conflicto), confiar en sí mismas, desinterés por estructuras jerárquicas, asumir retos centrados en el saber (dimensión técnica y conocimiento), estudio continuo y multiaprendizaje (leer diferentes áreas para saber hacer y adquirir mayor destreza en conocimientos), olvidar fácilmente situaciones de dolor, automotivarse para saber estar (buscar una motivación para salir adelante); asimismo, con capacidad de adaptación (potencial para aprender), perseverancia, gestión de emociones, dirección de personal, cohesión en equipos, asumir responsabilidades, planeación, influencia en otros y generación de estrategias para la solución de conflictos. Sin embargo, un alto porcentaje (70 \%) manifiesta no tener una óptima comunicación asertiva en todas las situaciones. Las habilidades (capacidad para realizar) en mujeres resilientes fueron pensamiento creativo, manejo de estrés y autoconocimiento.

De forma general, los relatos señalan un perfil de mujeres resilientes en busca de bienestar y felicidad para sí mismas y para sus familias, creativas, con alta importancia al logro personal, con creencias espirituales, marcadas por experiencias de vida que les permiten valorar cada experiencia ya sea positiva o negativa y con claros objetivos que proyectar su actuar.

Un puntaje promedio total de 84.5 y desviación 12.7 p=0.041 para resiliencia total, para las variables de resiliencia, el alfa de Cronbach para ítems en subescalas osciló entre 0.32 y 0.87 . Los ítems con índice de frabilidad inferior a 0.70 fueron removidos del test antes de realizar el análisis. Por otra parte, a fin de evaluar normalidad de la distribución, se aplicó la prueba de Kolmogorov-Smirnov, de la cual resultó que los reactivos no cumplían con tal supuesto. Sin embargo, se tomaron como criterio los parámetros de asimetría \pm 2 y curtosis \pm 7 en los que se puede considerar un comportamiento semejante al normal (Clark \& Watson, 1995). Los ítems 4, 11, 13, 15, 16, 17, 20, 21, 31 y 32 superaron estos valores reactivos descartándose al causar error de especificación en los modelos. Eliminados los reactivos que no cumplían con el supuesto de normalidad, se evaluaron dos modelos previos: en el primero resultaron dos dimensiones que explicaban el $62 \%$ de la varianza $\left(\mathrm{KMO}=0.92, \mathrm{p}<0.001\right.$, comunalidades $\left.>0.50, \mathrm{X}_{2}=3201.007, \mathrm{gl}=325\right)$. Para mejorar el ajuste se eliminaron los reactivos 30, 18, 9, 28 y 24, al contar con cargas factoriales menores a 0.50 , consideradas de aporte mínimo; y del segundo modelo resultante, de cuatro dimensiones que explicaban el $60 \%$ de la varianza $(K M O=0.91$, $<<0.001$, comunalidades 
$>0.50, \mathrm{X}^{2}=2516.954, \mathrm{gl}=148$ ), se eliminaron los ítems 5, 22, 27 y 30 por error de especificación, así como el reactivo 14 por criterio número mínimo de elementos para conformar una dimensión. Dado que los factores 1 y 3 mostraron una correlación alta, se llevó a cabo un análisis de componentes principales con los ítems involucrados. Mediante los resultados se corroboró que se trata de dos factores no diferenciados.

El modelo factorial final, con un índice $\mathrm{KMO}=0.90$ y prueba de esfericidad de Bartlett significativa $\left(\mathrm{X}^{2}=1671.113, \mathrm{gl}=148, \mathrm{p}<0.001\right)$, con comunalidades superiores a 0.50 , conformó dos dimensiones que explican el $64 \%$ de la varianza. Los autovalores encontrados van de 6.8 a 1. La carga factorial menor de los reactivos fue de 0.50 y la mayor de 0.77 .

En la tabla 1 se aprecia que los once ítems que forman el primer componente son parte de dimensiones denominadas percepción de sí mismo, futuro planeado y estilo estructurado. Se decidió renombrar el factor bajo el nombre planificación; mientras que el segundo factor, con cuatro ítems, se asoció a competencia social, recursos sociales y relaciones familiares, renombrándolo como empatía. La comparación de índices de bondad de ajuste absoluto muestra CMIN = 304.879, DF (130), GFI 0.92, AGFI=0.911, RMR=0.034, RM$\mathrm{SEA}=0.051 \mathrm{e}$ indices de bondad de ajuste $\mathrm{NFI}=0.9, \mathrm{TLI}=0.92, \mathrm{CFI}=0.93, \mathrm{IFI}=0.91, \mathrm{AIC}=313.879$, $\mathrm{BIC}=498.998$.

Los resultados para el cuestionario de fortaleza de carácter indican un alfa de 0.91 para curiosidad, o.88 para amor al conocimiento, 0.91 para mente abierta, 0.9 para creatividad, 0.89 para perspectiva, 0.75 para valentía, 0.9 para perseverancia, 0.7 para vitalidad, o.8 para amor, 0.9 para bondad, 0.89 para inteligencia social, 0.7 para civismo, 0.86 para equidad, 0.9 para liderazgo, 0.69 para humildad, o.6 para prudencia, o.8 para autorregulación, 0.87 para gratitud, 0.7 para esperanza, 0.54 para humor, 0.7 para espiritualidad, 0.8 para apreciación de la belleza y 0.9 para perdonar. 


\section{Tabla 1}

Matriz de estructura factorial obtenida para el cuestionario de resiliencia para mujeres universitarias en Norte de Santander

\begin{tabular}{lcc}
\hline \multicolumn{1}{c}{ RE } & F1 & F2 \\
\hline RE6 & 0.82 & \\
RE19 & 0.77 & \\
RE8 & 0.75 & \\
RE12 & 0.68 & \\
RE14 & 0.66 & \\
RE25 & 0.65 & \\
RE29 & 0.64 & \\
RE18 & 0.63 & \\
RE2 & 0.62 & \\
RE7 & 0.61 & \\
RE1 & 0.60 & \\
RE10 & & 0.74 \\
RE3 & & 0.71 \\
RE23 & & 0.66 \\
RE26 & & 0.63 \\
No de reactivos & 11 & 4 \\
Alfa de Cronbach & 0.81 & 0.9 \\
Porcentaje de & 33.8 & 30.2 \\
varianza explicada & & \\
\hline
\end{tabular}

En el análisis factorial la solución se agrupó en dos factores que explicarían el 69.95\% de la varianza. El factor I está formado por categorías creatividad $\mathrm{C}_{1}$ (media= $4.5 \mathrm{y}$ desviación estándar 0.4), mentalidad abierta $\mathrm{C}_{2}($ media $=4.54$ y desviación estándar 0.34), $\mathrm{C}_{3}$ curiosidad (media $=4.61 \mathrm{y}$ desviación estándar 0.48$)$, perspectiva $\mathrm{C}_{4}($ media $=4.45 \mathrm{y}$ desviación estándar 0.4), perseverancia $\mathrm{C}_{5}($ media = 4.1 y desviación estándar 0.54), liderazgo $\mathrm{C} 6$ (media $=4.35$ y desviación estándar 0.22$)$, honestidad $\mathrm{C}_{7}($ media $=4.5 \mathrm{y}$ desvia ción estándar 0.4), amor al conocimiento C8 (media= 4.61 y desviación estándar 0.64), apreciación de la belleza C9 (media $=4.5^{2}$ y desviación estándar 0.25 ), el factor II formado por inteligencia social $\mathrm{C}_{10}$ (media $=4.15 \mathrm{y}$ desviación estándar 0.65), valentía C11 (media $=4.5$ y desviación estándar 0.4 ), vitalidad $\mathrm{C}_{12}$ (media= 4.45 y desviación estándar 0.32), amor $\mathrm{C}_{13}\left(\right.$ media = $4.17 \mathrm{y}$ desviación estándar 0.49), bondad $\mathrm{C}_{14}($ media $=4.24 \mathrm{y}$ desviación estándar 0.4), civismo $\mathrm{C}_{15}($ media $=4.02 \mathrm{y}$ desviación estándar 0.76$)$, humildad $\mathrm{C} 16$ (media $=4.05 \mathrm{y}$ desviación estándar 0.74), prudencia $\mathrm{C}_{17}$ (media $=3.5 \mathrm{y}$ desviación estándar 0.94), humor C18 (media= 3.65 y desviación estándar 0.87), espiritualidad C19 
(media $=3.45$ y desviación estándar 0.68), perdonar C20 (media= 4.5 y desviación estándar o.3). El factor I se denomina conocimiento y sabiduría, mientras que el factor II humanidad. Implementado el cuestionario, en el modelo original los índices de ajuste fueron adecuados (CFI = 0.993; RMSEA = 0.051, WRMR = 0.710), pero los ítems se distribuyeron de forma irregular, los ISF <0.50, es decir, bajos (Domínguez \& Rodríguez, 2017) pese a que las correlaciones interfactoriales fueron moderadas.

La validación convergente se llevó a cabo al correlacionar las dimensiones del cuestionario de resiliencia ajustado para dos factores 15 ítems con la escala fortaleza de carácter, donde se obtuvieron correlaciones significativas en el nivel o1 para todas las dimensiones. No obstante, los ISF son muy bajos (<0 .70) en C11, C18, C19, pese a correlaciones interfactoriales moderadas (tabla 2), lo que indicaría que estos ítems no son influidos predominantemente por su factor teórico.

\section{Tabla 2}

Matriz de correlaciones entre resiliencia ajustada y fortaleza de carácter como medidas de validez convergente $y$ divergente

\begin{tabular}{lccc}
\hline $\begin{array}{c}\text { Ítem escala } \\
\text { fortaleza }\end{array}$ & $\begin{array}{c}\text { F1 } \\
\text { planificación }\end{array}$ & $\begin{array}{c}\text { F2 } \\
\text { empatía }\end{array}$ & ISF \\
\hline C1 & 0.80 & 0.71 & 0.98 \\
C2 & 0.79 & 0.82 & 0.97 \\
C3 & 0.80 & 0.72 & 0.84 \\
C4 & 0.92 & 0.68 & 0.96 \\
C5 & 0.89 & 0.61 & 0.99 \\
C6 & 0.85 & 0.78 & 0.88 \\
C7 & 0.69 & 0.79 & 0.84 \\
C8 & 0.91 & 0.63 & 0.76 \\
C9 & 0.67 & 0.61 & 0.53 \\
C10 & 0.85 & 0.89 & 0.89 \\
C11 & 0.49 & 0.58 & 0.51 \\
C12 & 0.71 & 0.84 & 0.90 \\
C13 & 0.72 & 0.82 & 0.93 \\
C14 & 0.41 & 0.72 & 0.81 \\
C15 & 0.85 & 0.92 & 0.94 \\
C16 & 0.92 & 0.93 & 0.96 \\
C17 & -0.69 & 0.35 & 0.81 \\
C18 & 0.06 & 0.14 & 0.49 \\
C19 & 0.18 & 0.48 & 0.53 \\
C20 & 0.41 & 0.61 & 0.81 \\
\hline
\end{tabular}


En el modelo bifactor ( $\mathrm{CFI}=0.948$; RMSEA=0.037; WRMR=0.622) los índices de ajuste favorables, no influidos predominantemente por su factor teórico, el $78 \%$ de cargas factoriales poseen un $\lambda$ mayor a 0.46 , confiabilidad de constructo $\omega=0.877, E C V=0.891$, $\mathrm{PUC}=0.784, \omega \mathrm{h}=0.90$, así como omega jerárquico por dimensiones $\omega \mathrm{hs}>0.141$. Una vez se realizó el modelamiento del conjunto de los ítems se aprecia que los constructos evaluados fueron los esperados, las cargas factoriales son de magnitud aceptable y el ISF es elevado. Estos aspectos previos respaldan los hallazgos que brindan evidencias de validez a la escala de resiliencia ajustada, la cual presentó una asociación positiva con fortaleza de carácter. La magnitud del coeficiente $\mathbf{a}$ hallado para los quince ítems teniendo en cuenta asociación entre los ítems ( $0.4<\mathrm{r}<0.81)$ consistente; así mismo, las magnitudes de los coeficientes enfocados en el constructo ( $\mathrm{H}$ y $\omega>0.87$ ), lo que da cuenta de la adecuada representación de la variable latente. Entonces, se puede concluir parcialmente que la confrabilidad de las puntuaciones y del constructo del test de resiliencia ajustado para mujeres universitarias en Norte de Santander es adecuada.

\section{Discusión}

La escala de resiliencia propuesta responde a la hipótesis de investigación planteada, pues las mujeres de Norte de Santander que estudian posgrado constituyen una población con características específicas que las hacen diferentes de otros conglomerados. Son mujeres creativas con alto nivel de resiliencia que tienen presente su familia y sus relaciones familiares, así como diferentes aspectos sociales ante la superación de diferentes adversidades que se les presentan; asimismo, capaces de asumir liderazgo y con alta fortaleza de carácter. La escala puede extrapolarse a poblaciones conformadas por mujeres que guarden un perfil similar a la de este estudio.

En autores de psicología social como Mattelart (2003) se considera que la actitud es la responsable de conducir el comportamiento; en algunos casos se establecen relaciones congruentes, mientras que en otros se identifican diferencias considerables en cuanto a lo que alguien cree y lo que termina haciendo una vez que se sumerge en una determinada situación social. Caso diferente se evidencia en Norte de Santander, donde las características de las mujeres que estudian posgrado en universidades públicas con alto nivel de resiliencia se centran en el saber vivir y en la calidad de vida centrada en la familia, así como 
en conocer sus fortalezas y controlar de sus debilidades; son mujeres creativas, que valoran la experiencia, proyectan su actuar y buscan el bienestar para sí y para sus familias.

Sobre el conocimiento y resiliencia en torno a su aplicación, relacionados con procesos de desarrollo identitario, al igual que Gil (2010), es pertinente expandir la resiliencia como proceso multidimensional y holístico; sacarla de los estrechos márgenes conceptuales que la circunscriben y la presentan como un constructo opuesto al riesgo, desarrollando nuevas metodologías que potencien su comprensión y el fortalecimiento del ser desde la gestión de factores incidentes en los procesos personales y socioafectivos que las mujeres profesionales llevan a cabo. Al respecto, similar a planteamientos de Rappaport (1981) se visibilizan las mujeres y en ellas su empoderamiento, concepto que ocupa un lugar primordial en la psicología comunitaria y en la intervención social (Zimmerman, 2000). El empoderamiento como factor se sustenta en las fortalezas, habilidades, competencias y sistemas de apoyo vital para la transformación de las comunidades (Montero, 2003). Este factor es importante en la resiliencia de las mujeres porque se lo relaciona con el desarrollo de potencialidades de su comportamiento, como son la identificación, el fomento de las capacidades, la promoción del bienestar y la identificación de factores de riesgo (Zimmerman, 2000), asociadas a posiciones de Scialpi (2003). Vanistendael (1995) coincide en señalar que el análisis de factores del enfoque de resiliencia en las mujeres aporta miradas optimistas, que permiten observar no solo brechas sino nuevos caminos para reconstruir políticas, realizar intervenciones sociales y mirar el rostro positivo de la realidad.

La escala propuesta y validada como instrumento difiere en el número de ítems y en la forma como aborda los diferentes factores de otras escalas como la de Connor y Davinson (2003); esta última aporta una medida de la capacidad de afrontar el estrés y consta de veinticinco ítems y define cinco factores a partir del análisis factorial resultante de su aplicación y validación, discriminando entre sujetos con mayor y menor resiliencia (Yárnoz-Yaben \& Comino-González, 2012). La escala propuesta aplica a mujeres profesionales y se compone de quince ítems desagregados en dos factores: once relacionados con el primer factor llamado planificación (asociado con la percepción de sí misma, la planeación del futuro y el estilo de vida estructurado) y cuatro con el segundo, denominado empatía, asociado a la competencia social, recursos sociales y relaciones familiares. Por otra parte, para evaluar la fortaleza de carácter se define una escala que involucra veinte ítems. Se encuentra alta correlación positiva entre las dimensiones del cuestionario de 
resiliencia y la escala de fortaleza de carácter. La validación de los conjuntos de ítems confirma que los constructos evaluados fue la esperada.

Como conclusión se puede establecer que las percepciones identificadas en mujeres en universidades se asocian a individualidades, cultura de machismo, ejemplo de vida y superación. Las características de las mujeres con alto nivel de resiliencia se centraron en el saber vivir con categoría primaria calidad de vida, en el saber, en saber hacer, adquirir mayor destreza en conocimientos y automotivarse para saber estar. Mujeres con capacidad de adaptación, perseverancia, gestión de emociones, dirección de personal, cohesión en equipos, asumir responsabilidades, planeación, influencia en otros, generación de estrategias para la solución de conflictos, habilidades en pensamiento creativo, manejo de estrés y autoconocimiento.

El perfil de mujeres resilientes se centra en búsqueda de bienestar y felicidad para sí mismas y para sus familias, creativas, con alta importancia al logro personal, marcadas por experiencias de vida que les permiten valorar cada experiencia - ya sea positiva o negativa- y claros objetivos que proyectan su actuar.

La escala de resiliencia ajustada a once ítems con dimensiones planificación y empatía orientada a mujeres universitarias en Norte de Santander es una medida de resiliencia de carácter unidimensional que presenta evidencias de validez con relación a su estructura interna, correlacionada con ítems de escala de fortaleza de carácter centrados en el dominio de un saber y en variables asociadas a humanidad, así como indicadores satisfactorios de confiabilidad del constructo y de sus puntuaciones.

\section{Referencias}

Aguilar, S. L., Gallegos, A. \& Muñoz, S. (2019). Análisis de componentes y definición del

concepto resiliencia: una revisión narrativa. Revista de Investigación Psicológica, 22, 77-100. American Educational Research Association, American Psychological Association \& National Council on Measurement in Education. (2014). Standards for Educational and Psychological Testing. American Educational Research Association.

Asparouhov, T., \& Muthén, B. (2009). Exploratory structural equation modeling. Structural Equation Modeling, 16(3), 397-438. https://doi.org/cdqfvc 
Avendaño, B., Avendaño, G., Cruz, W., \& Cárdenas, A. (2014). Guía de referencia para investigadores no expertos en el uso de estadística multivariada. Diversitas, 10(1), 13-27. https://doi.org/10.15332/s1794-9998.2014.0001.01

Baek, H. S., Lee, K. U., Joo, E. J., Lee, M. Y., \& Choi, K. S. (2010). Reliability and validity of the Korean version of the Connor-Davidson Resilience Scale. Psychiatry Investigation, 7(2), 109-115. https://doi.org/10.4306/pi.2010.7.2.109

Belykh, A. (2018). Resiliencia e inteligencia emocional: conceptos complementarios para empoderar al estudiante. Revista Latinoamericana de Estudios Educativos, 48(1), 255-282. https://doi.org/10.48102/rlee.2018.48.1.81

Benavente, M., \& Quevedo, M. (2018). Resiliencia, bienestar psicológico y afrontamiento en universitarios atendiendo a variables de personalidad y enfermedad. Revista de Psicología y Educación, 13(2), 99-112. https://doi.org/10.23923/rpye2018.01.161

Bender, A., \& Ingram, R. (2018). Connecting attachment style to resilience: Contributions of self-care and self-efficacy. Personality and Individual Differences, 130, 18-20. https:// doi.org/10.1016/j.paid.2018.03.038

Burns, R. A., \& Anstey, K. J. (2010). The Connor Davidson resilience scale (CD-Risc): Testing the invariance of a unidimensional resilience measure that is independent of positive and negative affect. Personality and Individual Differences, 48(5), 527-531. https://doi.org/10.1016/j.paid.2009.11.026

Cassidy, S. (2015). Resilience building in students: The role of academic self-efficacy. Frontiers in Psychology, 6, 17-81. https://doi.org/10.3389/fpsyg.2015.01781

Cicchetti, D., \& Rogosch, F. A. (2012). Gene x environment interaction and resilience: Effects of child maltreatment and serotonin, corticotropin releasing hormone, dopamine, and oxytocin genes. Development and Psychopathology, 24(2), 411-427. https://doi.org/10.1017/s0954579412000077

Clark, L., \& Watson, D. (1995). Constructing validity: Basic issues in objective scale development. Psychological Assessment, 7(3), 309. https://doi.org/10.1037/1040-3590.7.3.309

Cohen, J. (1992). A power primer. Psychological Bulletin, 112(1), 155-159. https://doi.org/as7

Connor, K. M., \& Davidson, J. R. (2003). Development of a new resilience scale: The Connor-Davidson Resilience Scale (CD-Risc). Depression and Anxiety, 18(2), 76-82. https://doi.org/10.1002/da.10113

Crespín, E. (2016). Análisis multivariante: aplicaciones con SPSS. Instituto de Ciencia, Tecnología e Innovación. 
Crespo, M., Fernández-Lansac, V., \& Soberón, C. (2014). Adaptación española de la escala de resiliencia de Connor-Davidson (CD-Risc) en situaciones de estrés crónico. Psicología Conductual, 22(2), 219-238.

Chen, F. (2007). Sensitivity of goodness of fit indexes to lack of measurement invariance. Structural Equation Modeling, 14(3), 464-504. https://doi.org/10.1080/10705510701301834

DeRosier, M. E., Frank, E., Schwartz, V., \& Leary, K. A. (2013). The potential role of resilience education for preventing mental health problems for college students. Psychiatric Annals, 43(12), 538-544. https://doi.org/10.3928/00485713-20131206-05

DiStefano, C., Liu, J., Jiang, N., \& Shi, D. (2018). Examination of the weighted root mean square residual: Evidence for trustworthiness? Structural Equation Modeling: A Multidisciplinary Journal, 25(3), 453-466. https://doi.org/10.1080/10705511.2017.1390394

Domínguez-Lara, S. (2016) Valores normativos de una escala de autoeficacia académica en estudiantes universitarios de Lima. Interacciones, 2(2), 91-98. https://doi.org/gn68

Domínguez-Lara, S. (2018). Propuesta de puntos de corte para cargas factoriales: una perspectiva de fiabilidad de constructo. Enfermería Clínica, 28(6), 401-402. https:// doi.org/10.1016/j.enfcli.2018.06.002

Domínguez-Lara, S., \& Rodríguez, A. (2017). Índices estadísticos de modelos bifactor. Interacciones, 3(2), 59-65. https://doi.org/10.24016/2017.v3n2.51

Escobedo, M., Hernández, J., Estebané, V., \& Martínez, G. (2015). Modelos de ecuaciones estructurales: características, fases, construcción, aplicación y resultados. Ciencia \& Trabajo, 18(55), 16-22. https://doi.org/10.4067/s0718-24492016000100004

Fleming, J., \& Merino, C. (2005). Medidas de simplicidad y ajuste factorial: un enfoque para la construcción y revisión de escalas derivadas factorialmente. Revista de Psicología, 23(2), 252-266. https://doi.org/10.1880o/psico.200502.002

Fu, C., Leoutsakos, J. M., \& Underwood, C. (2014). An examination of resilience crossculturally in child and adolescent survivors of the 2008 China earthquake using the Connor-Davidson Resilience Scale (CD-Risc). Journal of Affective Disorders, 155, 149-153. https://doi.org/10.1016/j.jad.2013.10.041

Gallardo-Pérez, H. de J., Vergel-Ortega, M., \& Villamizar, F. (2018). Investigación intervención y enfoque multimétodo en ciencias humanas y educación matemática. Logos, Ciencia y Tecnología, 9(2), 84-96. https://doi.org/10.22335/rlct.vgi2.458

García-Portilla, M. P., Bascarán, M. T., Sáiz, P. A., Parrallada, M., Bousoño, M., \& Bobes, J. (2017). Banco de instrumentos básicos de psiquiatría clínica. Cyesan. 
García-Vega, M., \& Domínguez-de la Ossa, E. (2013). Desarrollo teórico de la resiliencia y su aplicación en situaciones adversas: una revisión analítica. Revista Latinoamericana de Ciencias Sociales, Niñez y Juventud, 11(1), 63-77.

Garmezy, N. (1991). Resiliency and vulnerability to adverse developmental outcomes associated with poverty. American Behavioral Scientist, 34(4), 416-430. https://doi.org/cxm3kt

Gil, G. E. (2010). Los procesos holísticos de resiliencia en el desarrollo de identidades autorreferenciadas en Lesbianas, gays y bisexuales. Universidad de Las Palmas de Gran Canaria. Henderson, E. (2016). La resiliencia en el mundo de hoy. Gedisa.

Henderson, N., \& Milstein, M. (2003). Resiliencia en la escuela. Paidós.

Khoshouei, M. S. (2009). Psychometric evaluation of the Connor-Davidson resilience scale (CD-Risc) using Iranian students. International Journal of Testing, 9(1), 6o-66. https:// doi.org/10.1080/15305050902733471

Manzano, G. \& Ayala, G. C. (2013). Psychometric properties of Connor-Davidson Resilience Scale in a Spanish sample of entrepreneurs. Psicothema, 25(2), 245-251. https:// doi.org/10.7334/psicothema2012.183

Martínez, J., Vergel-Ortega, M., \& Zafra S. (2016). Comportamiento juvenil y competencias pro-sociales. Editorial Ibáñez.

Mattelart, A. (2003). Interview with Armand Mattelart. Information society and intellectual project. Sign and Thought Magazine, 22(43), 71-77.

McDonald, R. P., \& Ho, M. H. R. (2002). Principles and practice in reporting structural equation analyses. Psychological Methods, $7(1), 64-82$. https://doi.org/d4rsrf

Montero, M. (2003). Teoría y práctica de la psicología comunitaria. Paidós.

Ponce, F. P. (2015). Análisis exploratorio de modelos de ecuaciones estructurales sobre la escala Connor-Davidson (CD-Risc) en Chile y España. Salud \& Sociedad, 6(3), 238-247. https://doi.org/10.22199/So7187475.2015.0003.00004

Rappaport, J. (1981). In praise of paradox: A social policy of empowerment over prevention. American Journal of Community Psychology, 9(1), 1-21. https://doi.org/c426bj

Ruiz, M., Pardo, A., \& San Martín, M. (2010). Modelos de ecuaciones estructurales. Papeles del Psicólogo, 31(1), 34-45.

Salazar, J. P., \& Vergel-Ortega, M. (2018). Hermeneusis de la práctica pedagógica y formación de estudiantes matemáticamente competentes. En Investigación y praxis en la enseñanza de las matemáticas (71-104). Universidad Simón Bolívar.

Sameroff, A. J., \& Rosenblum, K. L. (2006). Psychosocial constraints on the development of resilience. Annals of the New York Academy of Sciences, 1094(1), 116-124. https:// doi.org/10.1196/annals.1376.010 
Santiago, M., Vergel-Ortega, M., \& Gallardo-Pérez, H. de J. (2020). Resiliencia en estudiantes exitosos en matemáticas. Praxis \& Saber, 11(26), e9973. https://doi.org/gn7b

Saris, W. E., Satorra, A., \& Van der Veld, W. M. (2009). Testing structural equation modeling or detection of misspecifications? Structural Equation Modeling, 16(4), 561-582. https://doi.org/10.1080/10705510903203433

Scialpi, D. (2003). Violencias en la administración pública: casos y miradas para pensar la administración pública nacional como ámbito laboral. Catálogos.

Schmidt, C. (2008). Construcción de un cuestionario de emociones positivas en población entrerriana. Ridep, 26(2), 117-139

Smits, I. A. M., Timmerman, M. E., Barelds, D. P. H., \& Meijer, R. R. (2015). The Dutch symptom checklist-go-revised: Is the use of the subscales justified? European Journal of Psychological Assessment, 31(4), 263-271. https://doi.org/10.1027/1015-5759/aooo233

Vanistendael, S. (1995). Cómo crecer superando los percances: resiliencia capitalizar las fuerzas del individuo. International Catholic Child Bureau.

Vásquez, C., \& Hervás, G. (2008). Psicología positiva aplicada. Desclée de Brouwer.

Vásquez, C., Hervás, G., \& Ho, S. (2006). Intervenciones clínicas basadas en la psicología positiva: fundamentos y aplicaciones. Psicología Conductual, 14(3), 401-432.

Vergel-Ortega, M., Duarte, H., \& Martínez-Lozano, J. (2016) Desarrollo del pensamiento matemático en estudiantes de cálculo integral su relación con la planificación docente. Revista Cientifica, 3(23), 17-29. https://doi.org/10.14483/udistrital.jour.rc.2015.23.22 Vergel-Ortega, M., Gallardo-Pérez, H. de J., \& Martínez, J. (2014). Factores asociados al rendimiento académico en estadística de estudiantes de administración pública. Colección Pedagogía Iberoamericana.

Vergel-Ortega, M., Martínez, J., \& Ortega, S. (2018). Validity of microrubri, instrument to measure the development of competences in mathematics. Journal of Physics: Conference Series, 116o. https://doi.org/10.1088/1742-6596/1160/1/012025

Vergel-Ortega, M., Martínez, J., \& Zafra, S. (2015). Validez de instrumento para medir la calidad de vida en la juventud: VIHDA. Revista Logos Ciencia \& Tecnología, 7(1) 17-26. https://doi.org/10.22335/rlct.v7i1.206

Vergel-Ortega, M., Martínez, J., \& Zafra, L. (2018). Inclúyeme en la U: imaginario de universidad y calidad en la juventud en situación de discapacidad de la ciudad de Cúcuta. Grupo Editorial Ibáñez.

Vizoso-Gómez, C., \& Arias-Gundín, O. (2018). Resiliencia, optimismo y burnout académico en estudiantes universitarios. European Journal of Education and Psychology, 11(1), 47-59. https://doi.org/10.30552/ejep.v11i1.185 
Yárnoz-Yaben, S., \& Comino-González, P. (2012). Un instrumento para la evaluación del perdón en el ámbito del divorcio y la separación. International Journal of Psychology and Psychological Therapy, $12(1), 49-58$.

Zimmerman, M. (2000). Empowerment theory. En J. Rappaport, \& E. Seidman (Eds.), Handbook of community psychology. Kluwer

Zinbarg, R. E., Yovel, I., Revelle, W., \& McDonald, R. P. (2006). Estimating generalizability to a latent variable common to all of a scale's indicators: A comparison of estimators for $\omega$ h. Applied Psychological Measurement, zo(2), 121-144. https://doi.org/b7dhps 